1 Supporting Information

2 Kinetics of all-dry free radical polymerization under 3 nano-confinement

4 Yifan Cheng†, Alexandra Khlyustova†, Pengyu Chen, Rong Yang*

5 Chemical and Biomolecular Engineering, Cornell University, Ithaca, NY 14853, U.S.A.

6

7 TThese authors contribute equally to this work

8

$9 \quad{ }^{*}$ Communication author contact information:

10 ryang@cornell.edu 


\section{Derivation of $\boldsymbol{D} \boldsymbol{R}_{\text {pore }}$ using the weight method}

12 Here we discuss the calculation of $D R_{\text {pore }}$ and random errors introduced into the $D R_{\text {pore }}$ calculation due to the variabilities and uncertainties at various steps of the weight method. To start, $D R_{\text {pore }}$ was determined by converting the weight gain due to polymer growth during iCVD to its volume, followed by normalization by the total exposed area and deposition time:

$$
D R_{\text {pore }}=\frac{\Delta m}{t \cdot \rho_{\text {poly }} \cdot A_{\text {exp }}}
$$

where $\Delta m$ is the weight gain, $t$ the deposition time, $\rho_{\text {poly }}$ the density of the vapor-deposited polymer films (for pHEMA, $\rho_{\text {poly }}=1.274 \mathrm{~g} \mathrm{~cm}^{-3}$ ), 3,4 and $A_{\text {exp }}$ the total AAO membrane surface area exposed to the reactant molecules. $A_{\text {exp }}$ was calculated by summing the total area of the pore walls $\left(A_{\mathrm{pw}}\right)$ and the non-porous area of the AAO top surface $\left(A_{\mathrm{np}}\right)$.

$$
A_{\text {exp }}=A_{\mathrm{np}}+A_{\mathrm{pw}}
$$

$A_{\mathrm{np}}$ and $A_{\mathrm{pw}}$ were then derived as the following:

$$
\begin{aligned}
& A_{\mathrm{np}}=\left(1-\varphi_{\mathrm{s}}\right) \cdot A_{\mathrm{anod}} \\
& A_{\mathrm{pw}}=\pi \cdot L \cdot D \cdot \rho_{\mathrm{pore}} \cdot A_{\mathrm{anod}}
\end{aligned}
$$

where $\varphi_{\mathrm{s}}$ is the areal porosity (i.e. total area of pore opening over total anodized area), which was determined based on the top-down SEM images of pristine AAO membranes, analyzed using ImageJ; $A_{\text {anod }}$ is the anodized area, equal to the area of the $25 \mathrm{~mm}$ circular AAO membrane; $L$ is the length of the AAO pores, which was determined by measuring thickness of the AAO membranes using the cross-sectional SEM images of these membranes; $D$ is the diameter of the AAO pores, which was determined by analyzing the top-down SEM images of pristine AAO membranes using ImageJ; and $\rho_{\text {pore }}$ is the areal number density of pores in the AAO membranes, which was calculated using the following equation:

$$
\rho_{\text {pore }}=\frac{\varphi_{\mathrm{s}} \cdot A_{\mathrm{anod}}}{\pi \cdot(D / 2)^{2}} \cdot \frac{1}{A_{\mathrm{anod}}}=\frac{4 \varphi_{\mathrm{s}}}{\pi D^{2}}
$$

\section{Error propagation analysis on $D R_{\text {pore }}$}

Error propagation analysis was conducted by applying a common simplification - i.e. assuming variables are independent (no correlations between variables), which yields the variance formula: ${ }^{5}$ 


$$
\sigma_{f}=\sqrt{\left(\frac{\partial f}{\partial x}\right)^{2} \sigma_{x}^{2}+\left(\frac{\partial f}{\partial y}\right)^{2} \sigma_{y}^{2}+\left(\frac{\partial f}{\partial z}\right)^{2} \sigma_{z}^{2}+\cdots}
$$

33 In the analysis reported here, to account for the variability in AAO geometries we focused on the error (or uncertainty) in $\varphi_{\mathrm{s}}, L$, and $D$, as they are most prone to introduce error into the thickness calculation via weight method. To account for the variation introduced during the iCVD process, we focused on the rate of weight gain $\Delta m / t$, where $\Delta m$ is the weight gain on AAO membrane due to polymer growth during the iCVD process and $t$ is the deposition time to achieve that weight gain, which involved random errors that might have occurred during the iCVD procedures (e.g., reaction termination based on interferometer readings and timing of the deposition) as well as the weight gain measurement using an analytical balance. Again, all aforementioned variables are assumed to be independent variables. Estimates for their mean and standard deviations were obtained from measurements of at least three AAO membranes. That led to the values of $\varphi_{\mathrm{s}}=(38$ $\pm 3) \%, L=(57.6 \pm 1.7) \mu \mathrm{m}$, and $D=(211 \pm 12) \mathrm{nm}$, and $\Delta m / t=(0.52 \pm 0.02) \mathrm{mg} \mathrm{min}^{-1} . \rho_{\text {pore }}$ was calculated to be $(1.1 \pm 0.2) \times 10^{9} \mathrm{~cm}^{-2}$ for the AAO membranes using the average values of $\varphi_{\mathrm{S}}, L$, and $D$. The outcome of the error propagation analysis on the AAO geometric variables, weight gain, and deposition time are shown in Table S1.

Table S1. Summary of the results of the error propagation analysis

\begin{tabular}{clll}
\hline Key parameter & $\boldsymbol{\mu} \pm \boldsymbol{\sigma}$ & Unit & Relative Error $\left(=\frac{\boldsymbol{\sigma}}{\boldsymbol{\mu}} \times \mathbf{1 0 0} \%\right)$ \\
$A_{\exp }$ & $(2.3 \pm 0.1) \times 10^{3}$ & $\mathrm{~cm}^{2}$ & $6.4 \%$ \\
$\Delta m / t$ & $(5.2 \pm 0.2) \times 10^{-1}$ & $\mathrm{mg} \mathrm{min}^{-1}$ & $4.0 \%$ \\
$D R_{\text {pore }}$ & $1.79 \pm 0.14$ & $\mathrm{~nm} \mathrm{~min}^{-1}$ & $7.6 \%$ \\
\hline
\end{tabular}

\section{Validation of the weight method}

50 We evaluated the effect of non-conformal coating thickness (i.e. faster polymer growth on the top 51 surface of an AAO membrane than inside its nanopores) on the weight-method estimates of 52 deposition kinetics under nano-confinement. Since the internal nanopore walls of AAO 53 membranes account for more than $99.7 \%$ of the total exposed surface area, we expect the weight 54 gain attributable to polymer growth on the internal pore walls to far outweigh that on the external 55 surfaces (i.e. top and bottom surface). On this basis, the average coating thickness (and 56 deposition kinetics) determined by applying the weight method should predominantly reflect the 
average polymer growth under nano-confinement. In fact, the effect of potential non-conformal polymer growth on the calculated average coating thickness could be assessed by evaluating the weight gain from an extreme non-conformal case - a 100-nm-thick pHEMA formed on both the top and bottom surface of the AAO membrane (matching the growth rate on Si wafer). This assessment shows that the weight gain $(\sim 0.11 \mathrm{mg})$ contributed by these extremely non-conformal surface polymer layers would increase the total weight gain by at most $\sim(15 \pm 6) \%$. In reality, the degree of non-conformality was far lower than this hypothetical case, as indicated by SEM images of the top (Fig S4), thus further ensuring the minimal impact of potential non-conformality on the deposition kinetics determined using the weight method.

\section{Derivation of $N_{\text {pore }}$}

In this section, we will derive the equation for the average number of collisions, $N_{\text {pore }}$ [Eq (1)]. For clarity of this derivation, we will first derive the average number of collisions contributed by the 'stick' scenario (i.e. free radicals chemisorb onto the pore wall before they leave a nanopore) and the 'non-stick' scenario (i.e. free radicals leave the pore without reacting) separately, and then sum them together to arrive at $N_{\text {pore }}$

First, let us consider the contributions to $N_{\text {pore }}$ by the 'stick' scenario, $N_{\text {pore }}$ (the superscript 's' denotes 'stick'). Here, we again divide $N_{\text {pore }}$ into two parts: i) the contributions by the radicals that 'stick' upon $1^{\text {st }}, 2^{\text {nd }}, \cdots,\left(N_{\max }-1\right)^{\text {th }}$ collision with the pore wall - namely, $N_{\text {pore }}{ }^{\text {s1 }}$ and ii) $N_{\max }{ }^{\text {th }}$ collision - namely, $N_{\text {pore }}{ }^{\mathrm{s} 2}$. This is because for $N_{\text {pore }}{ }^{\mathrm{s} 1}$, the number of collisions does not depend on the depth of the first collision (see Fig S5A); whereas if $N_{\max }{ }^{\text {th }}$ collision occurs or not depends on where $1^{\text {st }}$ collision is along the pore length. The expectation of $N_{\text {pore }}{ }^{\text {s1 }}$ follows the Bernoulli distribution, which can be expressed as the following:

$$
N_{\text {pore }} s 1=\sum_{n=1}^{N_{\text {max }}-1} n \Gamma(1-\Gamma)^{n-1}
$$

Where $N_{\max }$ is calculated using the following equation:

$$
N_{\text {max }}=\text { Integer }\left[\frac{L}{D \cdot \tan \theta}\right]+1
$$

Among the free radicals that have collided with the pore wall $\left(N_{\max }-1\right)$ times without reacting, only a fraction of them (i.e. $\varepsilon / l_{0}$, the orange fraction in Fig S5B) can make the $N_{\text {max }}{ }^{\text {th }}$ collision and stick; the rest (i.e. $1-\varepsilon / l_{0}$, the gray fraction in Fig S5B) would overshoot. Therefore, the expectation of $N_{\text {pore }}$ s2 is:

$$
N_{\text {pore }} \mathrm{s}^{2}=\left(\varepsilon / l_{0}\right) \cdot\left[N_{\max } \Gamma(1-\Gamma)^{N_{\max }-1}\right]
$$


84 Where $\varepsilon$ is the residual length of the pore, and $l_{0}$ the step size. They are defined as the followings:

$$
\begin{aligned}
& l_{0}=D \cdot \tan \theta \\
& \varepsilon=L-\text { Integer }\left[L / l_{0}\right] \cdot l_{0}
\end{aligned}
$$

85 Thus, total contribution by the 'stick' scenario $\left(N_{\text {pore }}{ }^{\mathrm{s}}\right)$ can be calculated as the following:

$$
\begin{aligned}
N_{\text {pore }}{ }^{\mathrm{s}} & =N_{\text {pore }}{ }^{\mathrm{s} 1}+N_{\text {pore }} \mathrm{s}^{2} \\
& =\left(\varepsilon / l_{0}\right) \cdot\left[N_{\max } \Gamma(1-\Gamma)^{N_{\max }-1}\right]+\sum_{n=1}^{N_{\max }-1} n \Gamma(1-\Gamma)^{n-1}
\end{aligned}
$$

86 Next, let us consider the contributions to $N_{\text {pore }}$ by the 'non-stick' scenario, $N_{\text {pore }}{ }^{\text {ns }}$. As shown in

87 Fig S5B, a definite proportion of such radicals (i.e. $\varepsilon / l_{0}$ ) can make the $N_{\text {max }}^{\text {th }}$ collision with the pore wall right before they exit, whereas the remaining proportion (i.e. $1-\varepsilon / l_{0}$ ) overshoot the pore end, thus exiting with $\left(N_{\max }-1\right)$ collisions. The expected number of collisions from the 'nonstick' free radicals is therefore:

$$
N_{\text {pore }}{ }^{n s}=\left(\varepsilon / l_{0}\right) \cdot N_{\max }(1-\Gamma)^{N_{\max }}+\left(1-\varepsilon / l_{0}\right) \cdot\left[\left(N_{\max }-1\right)(1-\Gamma)^{N_{\max }-1}\right]
$$

91 Now, we combine the expected (or average) number of collisions contributed by the 'stick' and

92 'non-stick' cases by summing $N_{\text {pore }}$ s and $N_{\text {pore }}$ ns together. After some minor algebraic

93 manipulations, we get:

$$
\begin{aligned}
N_{\text {pore }} & =N_{\text {pore }} \mathrm{s}+N_{\text {pore }} \mathrm{ns}^{N_{\max ^{-1}}} n \Gamma(1-\Gamma)^{n-1} \\
& =\frac{L}{D \cdot \tan \theta}(1-\Gamma)^{N_{\max ^{-1}}+\sum_{n=1} n \Gamma}
\end{aligned}
$$

The first term of Eq (S14) [i.e. Eq (1)] describes the contributions to the total number of radicalsurface collisions by i) those radicals that never react with the surface-adsorbed monomers and end up exiting the pore (i.e. $N_{\text {pore }}{ }^{\mathrm{ns}}$ ) and ii) the radicals that chemisorb upon $N_{\max }{ }^{\text {th }}$ collision with the pore wall (i.e. $N_{\text {pore }}{ }^{\mathrm{s} 2}$ ). The purpose of $\frac{L}{D \cdot \tan \theta}$ of the first term is to account for the variation in the maximum collisions of those 'non-stick' radicals based on where along the pore length their first collision with the pore wall occurs. The second term (i.e. the finite series) accounts for the contributions by the radicals that 'stick' upon $1^{\text {st }}, 2^{\text {nd }}, \cdots,\left(N_{\max }-1\right)^{\text {th }}$ collision with the pore wall (i.e. $102 N_{\text {pore }}{ }^{\mathrm{s} 2}$ ).

\section{Derivation of $\boldsymbol{\eta}$}

The expectation for the number of free radical-surface collisions it takes for a free radical of 106 sticking probability $\Gamma$ to stick to the pore wall is $1 / \Gamma$. For such a radical, we define a critical incident 
angle, beyond which the radical leaves the pore (i.e. it travels farther than the pore length $L$ along pore length) before the radical-surface collision count reaches $1 / \Gamma$. Thus,

$$
\begin{aligned}
& D \cdot(1 / \Gamma) \cdot \tan \theta_{\mathrm{c}}=L \\
& \theta_{\mathrm{c}}=\arctan (\Gamma \cdot L / D)
\end{aligned}
$$

where $\theta_{c}$ is the critical incident angle, above which the incident free radical is expected to leave the pore without reacting.

Assuming an even distribution of incident angle $\theta \in(0, \pi / 2]$ for the free radicals that have entered the pore, we can estimate the proportion $(\eta)$ of those radicals that exit the pore without reacting (despite their numerous collisions with the pore wall) using the following equation:

$$
\eta=\frac{\pi / 2-\theta_{\mathrm{c}}}{\pi / 2}
$$

This concludes the derivation of $\eta$ in the main text.

\section{Finite element analysis of AAO membrane temperature}

To test the hypothesis that lower thermal conductivity of the AAO membrane resulted in a higher substrate temperature (than the iCVD stage and the Si wafer thereon), finite element analysis (FEA) was performed on the AAO membrane using COMSOL Multiphysics v5.4 (COMSOL Inc., Stockholm, Sweden). The physical model consists of two stacked layers: the top layer $(55 \mu \mathrm{m})$ corresponds to the membrane and the bottom layer $(5 \mu \mathrm{m})$ to a vapor layer. The $5-\mu \mathrm{m}$ thick vapor layer was set up because the peripheral of the membrane was held up by a polypropylene holder. This vertical offset disrupted the contact between the membrane and iCVD cooling stage. To simulate the heat transfer and thus the temperature profile of the membrane, we input the following values to the COMSOL model: the thermal conductivity of the vapor layer was set as $4.4 \times 10^{-5} \mathrm{~W}(\mathrm{~m} \mathrm{~K})^{-1}$. To account for disruption of heat conduction in radial directions by the embedded pores, the thermal conductivity of the AAO membrane was set to $0.95 \mathrm{~W}\left(\mathrm{~m} \mathrm{~K}^{-1}\right.$ in X$\mathrm{Y}$ direction and $1.6 \mathrm{~W}\left(\mathrm{~m} \mathrm{~K}^{-1}\right.$ in $\mathrm{Z}$ direction (i.e. along the pores) (see Figure S7). As for boundary conditions: the surrounding and bottom boundaries of the stack were set to $30^{\circ} \mathrm{C}$ due to direct contact with the stage. The top surface was heated by radiation from the filament array (set to $230^{\circ} \mathrm{C}$ ) while cooled by the $30^{\circ} \mathrm{C}$ vapor layer. Under these conditions, a bulk-average temperature of $36.1^{\circ} \mathrm{C}$ was obtained for the AAO membrane. This temperature discrepancy between the AAO membrane and Si wafer resulted in a $P_{\mathrm{M}, \mathrm{Si}}^{\mathrm{sat}} / P_{\mathrm{M}, \mathrm{AAO}}^{\mathrm{sat}} \sim 0.63$, which in turn contributed to the slower deposition rate observed with the AAO membrane. 


\section{Direct measurement of AAO membrane temperature}

136 To verify the simulation results above, we affixed a Type $K$ thermocouple directly onto the top 137 surface of the AAO membrane via a Kapton® tape and compared the reading from that 138 thermocouple with the stage temperature (set to $30{ }^{\circ} \mathrm{C}$ ). The readings were recorded using a 139 digital thermometer at equilibrium under typical deposition conditions (i.e. $T_{\text {stage }}=30{ }^{\circ} \mathrm{C}, T_{\text {filament }}$ $140=230{ }^{\circ} \mathrm{C}, P_{\text {reactor }}=350 \mathrm{mTorr}$, gas flow rate $\left.=0.68 \mathrm{sccm}\right)$. Temperature of the Si wafer was 141 assumed to be the same as that of the stage. The results showed that when the stage temperature 142 read $30.2{ }^{\circ} \mathrm{C}$, the AAO membrane temperature read $36.3^{\circ} \mathrm{C}$, which is in close agreement with the 143 temperature $\left(36 .{ }^{\circ} \mathrm{C}\right)$ predicted by the COMSOL model, thus adding weight to the $\sim{ }^{\circ} \mathrm{C}$ 144 temperature difference between the AAO membrane and the Si wafer. 
147 Figure S1. Surface topography of (A) AAO membranes (nominal pore diameter $=200 \mathrm{~nm}$ ) and 148 (B) Si wafers, characterized using atomic force microscopy (AFM). (C) and (D) showed height 149 variation along the dashed lines in $(A)$ and $(B)$, respectively.

150
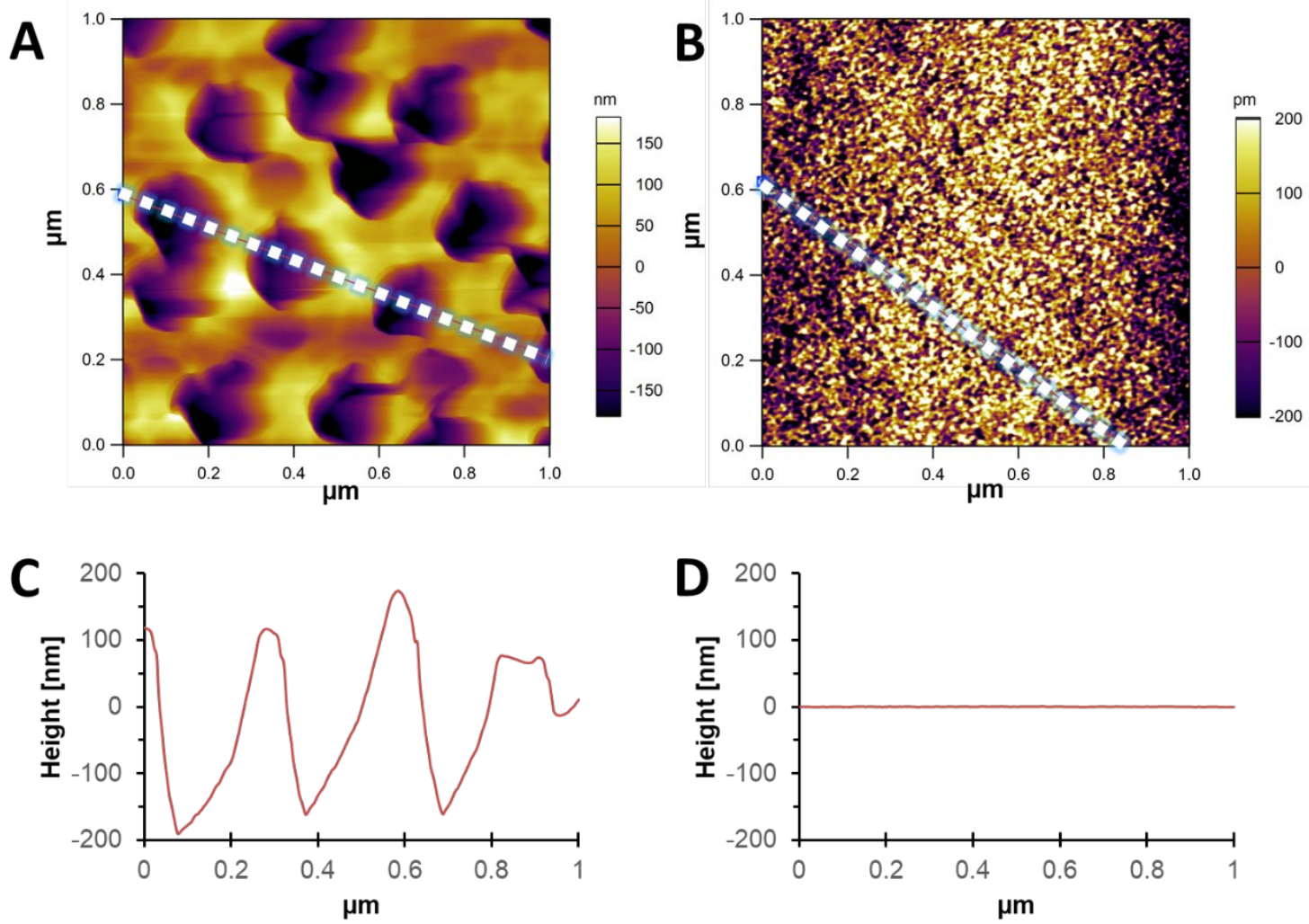

151 
153 Figure S2. SEM cross-sectional images showing the largely straight pores of AAO membranes 154 with minor irregularity within the first $500 \mathrm{~nm}$.

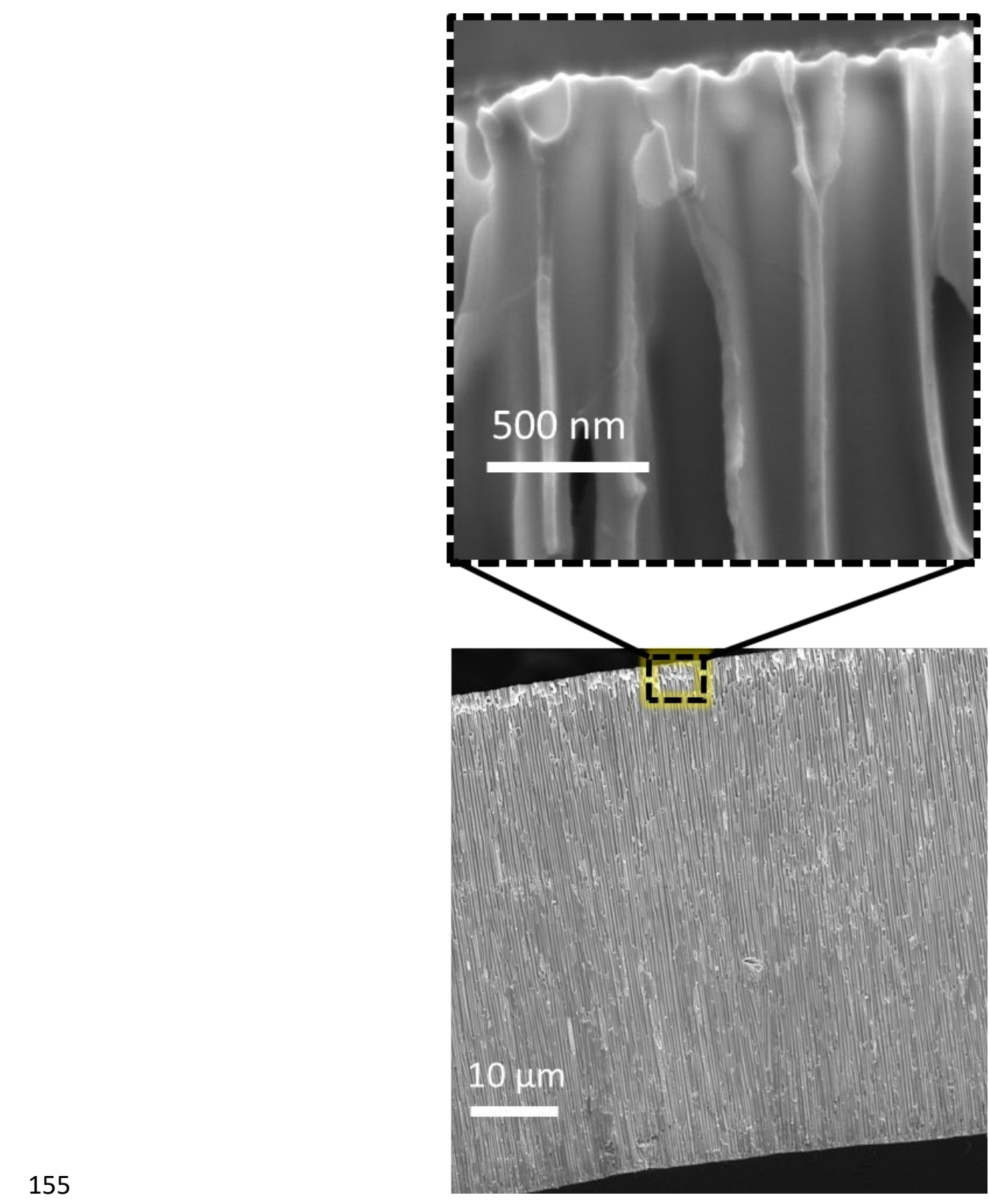

156 
157 Figure S3. (A-D) Representative cross-sectional SEM images acquired at various distances from 158 the top of the pHEMA-coated AAO membrane and $(\mathrm{E}-\mathrm{H})$ their corresponding zoom-in views 159 showing details of the pHEMA coatings. The white arrows in $(A)$ and $(E)$ denote the porous space 160 concealed by the draped thin film skin layer, possibly an artifact caused by breaking the 161 membrane.

\section{Distance from Top}

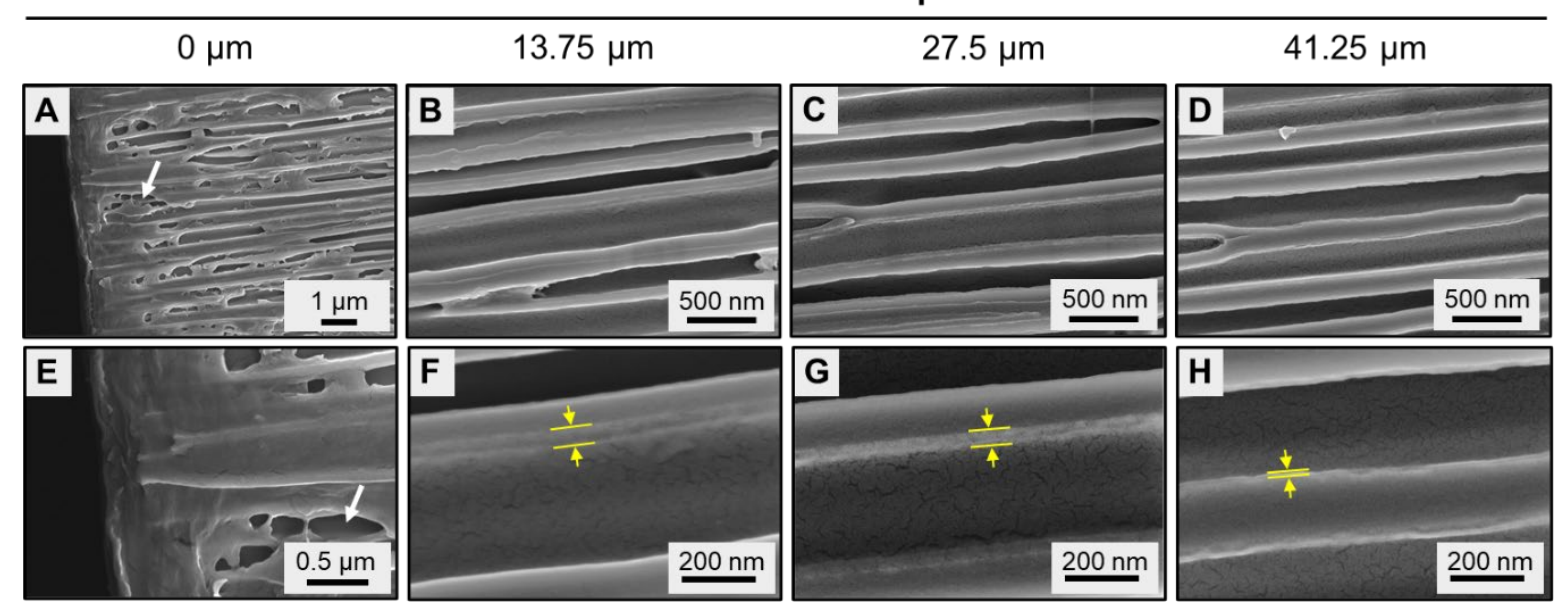


163 Figure S4. (A) SEM top-view image of a pristine AAO membrane. (B) Same AAO membrane 164 coated with pHEMA (100 nm on Si wafer) under the highest $P_{M} / P_{M}^{\text {sat }}$ (value $=0.56$ ) and the highest $165 P_{I} / P_{I}^{\text {sat }}($ value $=0.007)$ used in this work
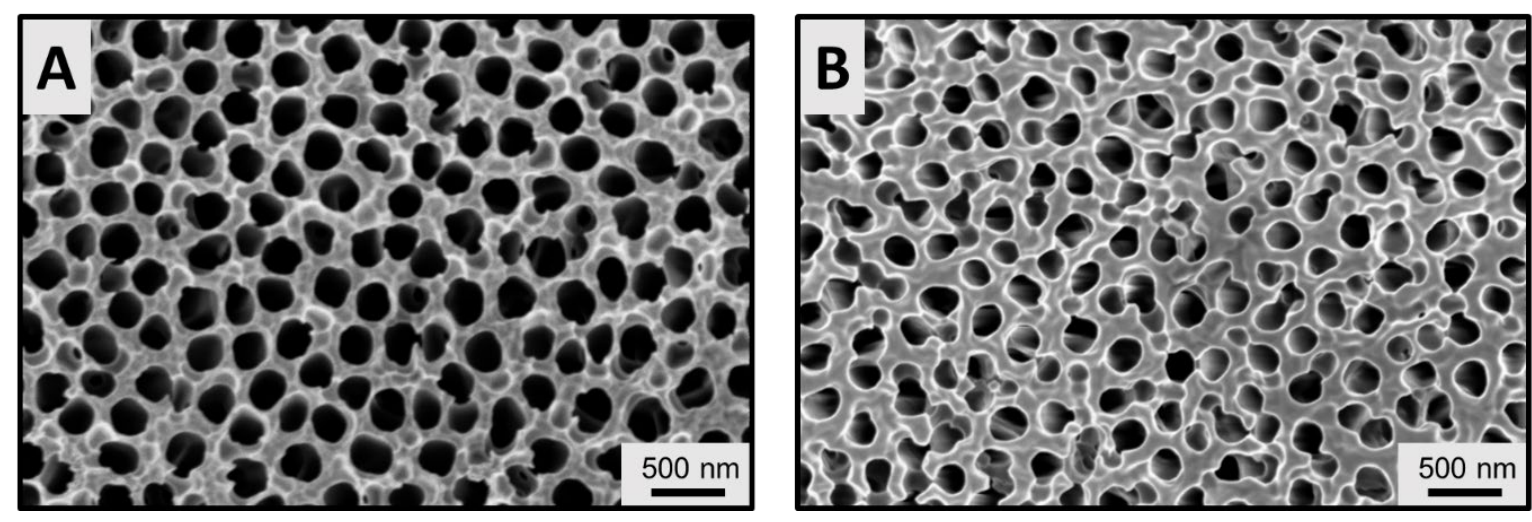
Figure S5. (A) Schematic of the mass transport of free radicals within a nanopore during 169 deposition (assuming specular reflection). The maximum number of radical-surface collisions 170 could be either $N_{\max }$ or $\left(N_{\max }-1\right)$, depending on the depth at which the first radical-surface collision 171 occurs. The orange arrowed lines represent the free radical 'upper bound' trajectory, while the 172 gray ones the 'lower bound' trajectory; (B) a zoom-in view at the bottom of the pore, showing the 173 relationship between the proportion of free radicals that can have the $N_{\max }{ }^{\text {th }}$ collision (represented 174 by the orange shaded area) and the key geometric parameters $\varepsilon$ and $l_{0}$.

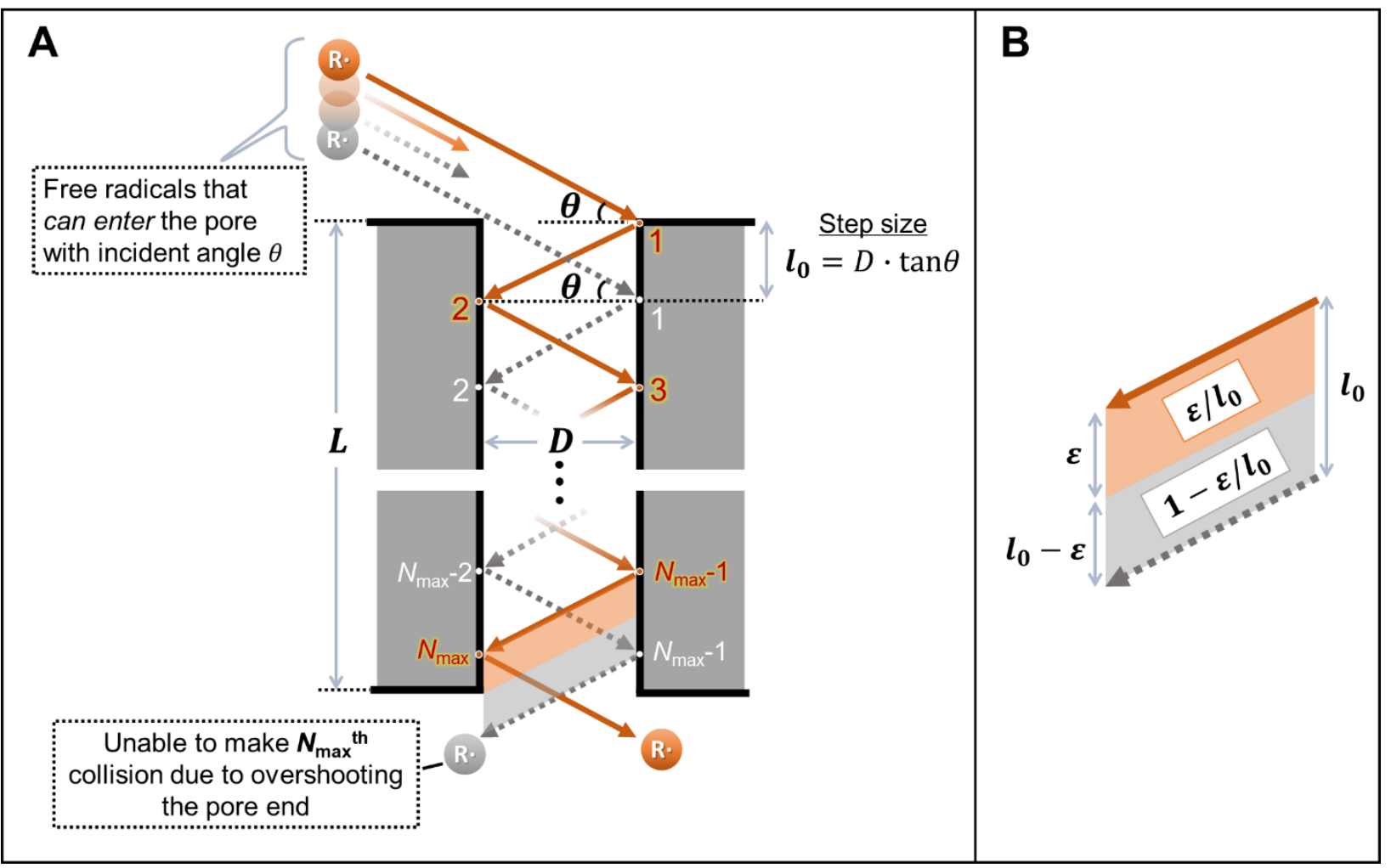


177 Figure S6. Model predicted relative areal collision frequency, $\beta$, as a function of pore diameter, $178 D$, at radical sticking probability, $\Gamma$, of $10^{-2}, 10^{-4}, 10^{-6}$, and $10^{-8}$. Pore length $L=55 \mu \mathrm{m}$ for all 179 calculations (Eq 5). Purple arrows indicate the AAO pores ( $D=200 \mathrm{~nm}$ ) used in this work. Inset 180 shows the data on a log scale.

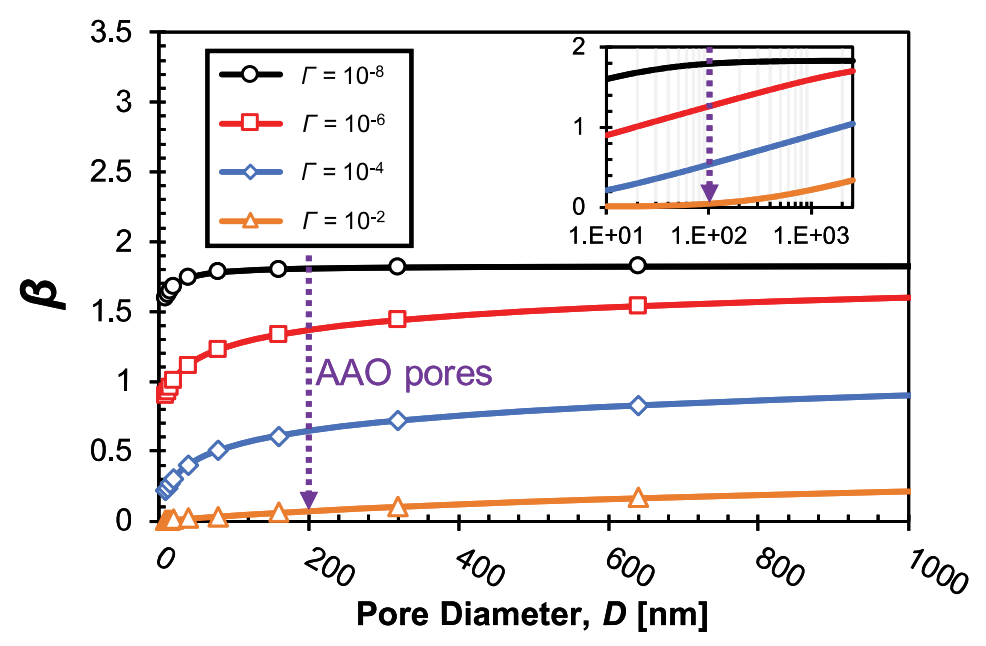

181 
183 Figure S7. Finite element analysis of the temperature profile of the AAO membranes under the 184 actual iCVD deposition conditions (i.e. $T_{\text {stage }}=30{ }^{\circ} \mathrm{C}, T_{\text {filament }}=230{ }^{\circ} \mathrm{C}, P_{\text {reactor }}=350 \mathrm{mTorr}$, gas 185 flow rate $=0.68 \mathrm{sccm})$.

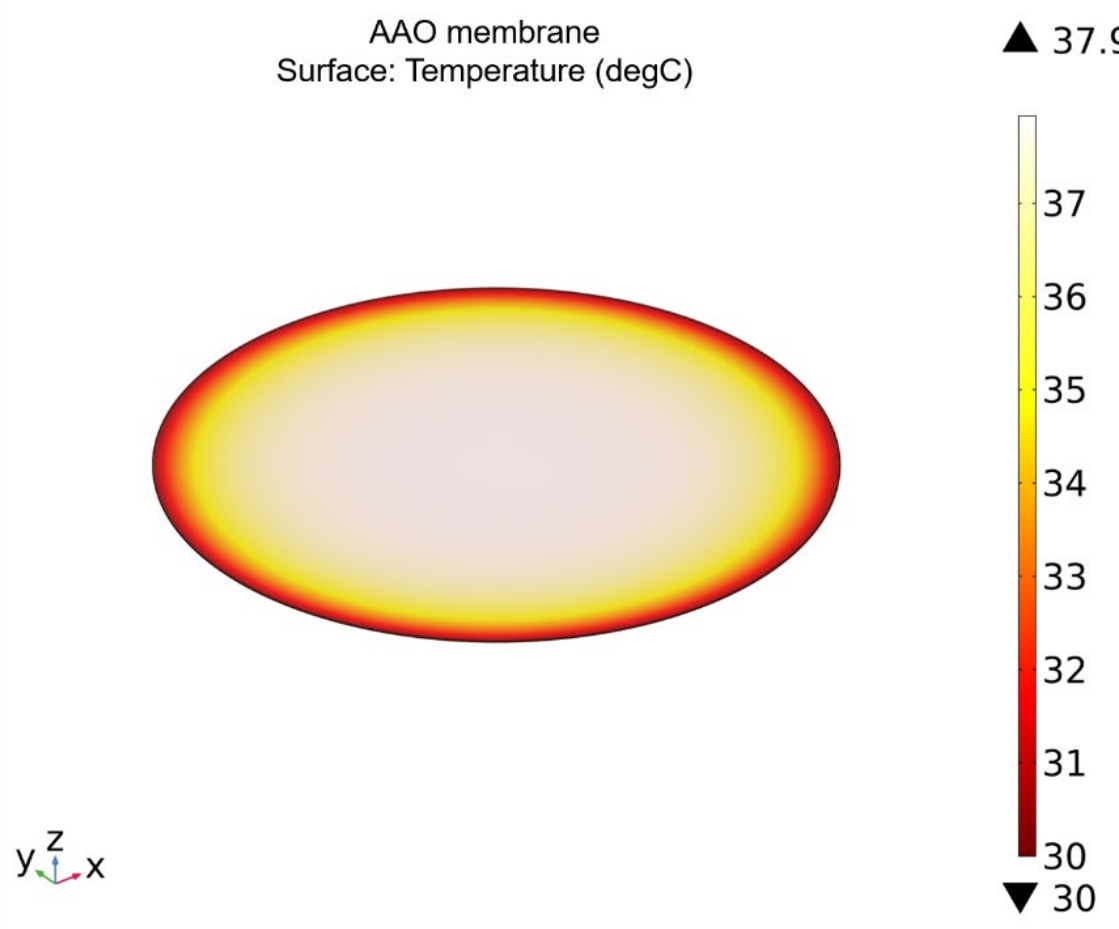

186

187 